Bull. Austral. Math. Soc.

VOL. 48 (1993) [435-440]

\title{
ON A RESONANCE PROBLEM WITH NONLINEARITIES OF ARBITRARY POLYNOMIAL GROWTH
}

\author{
Chung-Wei Ha and Wen-Bing Song
}

\begin{abstract}
We prove some existence theorems for solutions of a semilinear two point boundary value problem at resonance in which the nonlinear terms can have arbitrary polynomial growth in one of the directions $\infty$ or $-\infty$, and are bounded in the other. The results are based on degree theoretic arguments and the Borsuk odd mapping theorem.
\end{abstract}

We consider the boundary value problem

$$
u^{\prime \prime}+u+g(x, u)=h(x) \quad \text { in } \quad(0, \pi), \quad u(0)=u(\pi)=0
$$

where $h \in L^{1}(0, \pi)$ is given and $g:(0, \pi) \times \mathbb{R} \rightarrow \mathbb{R}$ is a Caratheodory function, that is, $g(x, u)$ is measurable in $x \in(0, \pi)$ for all $u \in \mathbb{R}$ and continuous in $u \in \mathbb{R}$ for almost all $x \in(0, \pi)$. The solvability of the problem (1) has been extensively studied if the nonlinear term $g$ is assumed to grow at most linearly in $u$. Let

$$
\Gamma_{+}(x)=\limsup _{u \rightarrow \infty} g(x, u) / u, \quad \Gamma_{-}(x)=\limsup _{u \rightarrow-\infty} g(x, u) / u
$$

be nonnegative functions in $L^{\infty}(0, \pi), \Gamma(x)=\max \left\{\Gamma_{+}(x), \Gamma_{-}(x)\right\}$. Existence theorems for a solution to (1) are proved if $\Gamma(x) \leqslant 3$ for almost all $x \in(0, \pi)$, with the strict inequality holding on a subset of $(0, \pi)$ of positive measure (see Ahmad [1], IannacciNkashama [4]). A further result along these lines is obtained in Drabek [3] in which the bound in $L^{\infty}(0, \pi)$ of one of $\Gamma_{+}$and $\Gamma_{-}$can be arbitrary, provided that of the other is sufficiently small. The purpose of this paper is to give solvability conditions for (1) when $g$ is allowed to have arbitrary polynomial growth in $u$ in one of the directions $\infty$ or $-\infty$, and is bounded in $L^{1}(0, \pi)$ in the other. Our results complement those cited above. For definiteness we assume that

(H) There exist constants $a, p \geqslant 0$ and $b \in L^{1}(0, \pi)$ such that $b \geqslant 0$ in $(0, \pi)$ and for almost all $x \in(0, \pi), u \in \mathbb{R}$

$$
-b(x) \leqslant g(x, u) \leqslant a|u|^{p}+b(x) .
$$

Received 10 December 1992

Work supported in part by National Science Council, ROC project 79-0208-M007-44.

Copyright Clearance Centre, Inc. Serial-fee code: 0004-9729/93 \$A2.00+0.00. 
Our main result is Theorem 1 below which is an existence theorem for a solution to (1) by assuming a Landesman-Lazer condition (see (3) below) originally obtained in Landesman and Lazer [5]. In Theorem 2 we give solvability conditions for (1) in the absence of a Landesman-Lazer condition. For their proofs, we use degree theoretic arguments (see, for example Deimling [2], Chapter 2) and the Borsuk odd mapping theorem.

In what follows, we shall make use of the real Banach spaces $L^{p}(0, \pi), C[0, \pi]$ and $C^{1}[0, \pi]$ with the standard norms denoted by $\|u\|_{L^{p}},\|u\|_{C}$ and $\|u\|_{C^{1}}$, respectively. By a solution of (1) we mean a function which has an absolutely continuous derivative and satisfies the boundary condition and the differential equation in (1) almost everywhere in $(0, \pi)$.

THEOREM 1. Let $g:(0, \pi) \times \mathbb{R} \rightarrow \mathbb{R}$ be a Caratheodory function satisfying the condition (H). If there exists $c \in L^{1}(0, \pi)$ such that for almost all $x \in(0, \pi), u \leqslant 0$

$$
g(x, u) \leqslant c(x),
$$

then for any $h \in L^{1}(0, \pi)$ the problem (1) is solvable provided that

$$
\int_{0}^{\pi} g_{-}(x) \sin x d x<\int_{0}^{\pi} h(x) \sin x d x<\int_{0}^{\pi} g_{+}(x) \sin x d x
$$

where $g_{+}(x)=\liminf _{u \rightarrow \infty} g(x, u), g_{-}(x)=\limsup _{u \rightarrow-\infty} g(x, u)$.

Proof: We can sssume without loss of generality that $p>1$. Let $f: \mathbb{R} \rightarrow \mathbb{R}$ be the continuous function defined by

$$
f(u)=\left\{\begin{array}{cl}
u & \text { if }|u| \leqslant 1 \\
1 & \text { if } u>1 \\
-1 & \text { if } u<-1
\end{array}\right.
$$

We consider the boundary value problems

$$
\begin{gathered}
u^{\prime \prime}+u+(1-t) f(u)+\operatorname{tg}(x, u)=\operatorname{th}(x) \text { in }(0, \pi) \\
u(0)=u(\pi)=0
\end{gathered}
$$

for $0 \leqslant t \leqslant 1$, which becomes the original problem (1) when $t=1$.

We assume for the moment that solutions $u$ to (4) for some $0 \leqslant t \leqslant 1$ have an a priori bound in $C^{1}[0, \pi]$ and use this to finish proving the theorem. Then there exists a constant $R>0$ such that $\|u\|_{C}<R$ for any possible solution $u$ to (4) for some $0 \leqslant t \leqslant 1$. We define a map $B:[0,1] \times C[0, \pi] \rightarrow C[0, \pi],(t, u) \rightarrow H_{t} u$, where $w=H_{t} u$ is the unique solution to the problem

$$
\begin{gathered}
w^{\prime \prime}+u+(1-t) f(u)+t g(x, u)=\operatorname{th}(x) \text { in }(0, \pi) \\
w(0)=w(\pi)=0 .
\end{gathered}
$$


Then a function $u \in C[0, \pi]$ is a solution to (4) for some $0 \leqslant t \leqslant 1$ if and only if $H_{t} u=$ $u$. It follows from a standard argument that $H$ is a compact map. Moreover, the LeraySchauder degree $\operatorname{deg}\left(I-H_{t}, B, 0\right)$ is defined and does not depend on $0 \leqslant t \leqslant 1$, where $I$ denotes the identity map on $C[0, \pi], B=\left\{u \in C[0, \pi]:\|u\|_{C}<R\right\}$. Obviously the map $H_{0}$ is odd on $C[0, \pi]$ and so by the Borsuk odd mapping theorem (see, for example Deimling [2], Theorem 8.3) $\operatorname{deg}\left(I-H_{0}, B, 0\right)$ is odd. Hence deg $\left(I-H_{1}, B, 0\right)$ is odd, which implies that the original problem (1) has at least a solution in $B$.

In order to prove the assumption, we need some preliminary results. First we note that there exist Caratheodory functions $g_{1}, g_{2}:(0, \pi) \times \mathbb{R} \rightarrow \mathbb{R}$ such that $g=g_{1}+g_{2}$ and $\left|g_{1}(x, u)\right| \leqslant b(x), 0 \leqslant g_{2}(x, u) \leqslant a|u|^{p}$ for almost all $x \in(0, \pi), u \in \mathbb{R}$. This may be done by defining

$$
g_{2}(x, u)=\min \left\{g(x, u)+b(x), a|u|^{p}\right\}
$$

and $g_{1}=g-g_{2}$. We note also the inequality

$$
|w(x) / \sin x| \leqslant(\pi / 2) \max _{0 \leqslant \bullet \leqslant x}\left|w^{\prime}(s)\right|
$$

for $x \in[0, \pi]$ valid for all $w \in C^{1}[0, \pi]$ with $w(0)=w(\pi)=0$. Now we suppose that $u$ is a solution to (4) for some $0 \leqslant t \leqslant 1$. Taking the inner product in $L^{2}(0, \pi)$ of (4) with $\sin x$, we have

$$
(1-t) \int_{0}^{\pi} f(u) \sin x d x+t \int_{0}^{\pi}[g(x, u)-h(x)] \sin x d x=0
$$

and so

$$
t \int_{0}^{\pi}\left|g_{2}(x, u)\right| \sin x d x \leqslant \int_{0}^{\pi}[1+b(x)+|h(x)|] \sin x d x \equiv c_{1} .
$$

It follows from (5) that

$$
\begin{aligned}
\int_{0}^{\pi}\left|\operatorname{tg}_{2}(x, u)\right|^{(1+p) / p} d x & \leqslant t a^{1 / p} \int_{0}^{\pi}\left|g_{2}(x, u)\right||u| d x \\
& \leqslant t a^{1 / p}(\pi / 2)\|u\|_{C^{1}} \int_{0}^{\pi}\left|g_{2}(x, u)\right| \sin x d x \\
& \leqslant c_{1} a^{1 / p}(\pi / 2)\|u\|_{C^{1}}
\end{aligned}
$$

Hence there exists a constant $c_{0}>0$ such that

$$
\begin{aligned}
t \int_{0}^{\pi}\left|g_{2}(x, u)\right| d x & \leqslant \pi^{1 /(1+p)}\left(\int_{0}^{\pi}\left|g_{2}(x, u)\right|^{(1+p) / p} d x\right)^{p /(1+p)} \\
& \leqslant c_{0}\|u\|_{C^{1}}^{p /(1+p)}
\end{aligned}
$$


for any possible solution $u$ to (4) for some $0 \leqslant t \leqslant 1$.

It remains to show that solutions $u$ to (4) for some $0 \leqslant t<1$ have an a priori bound in $C^{1}[0, \pi]$. If this is false, then there exist a sequence $\left\{t_{n}\right\}$ in $[0,1)$ and a corresponding sequence $\left\{u_{n}\right\}$ of functions such that $u_{n}$ is a solution to (4) when $t=t_{n}$ and $\left\|u_{n}\right\|_{C^{1}} \geqslant n$ for all $n$. Let $v_{n}=u_{n} /\left\|u_{n}\right\|_{C^{1}}$. Then $\left\|v_{n}\right\|_{C^{1}}=1$ and

$$
v_{n}^{\prime \prime}+v_{n}+m_{n}=0 \quad \text { in }(0, \pi), \quad v_{n}(0)=v_{n}(\pi)=0,
$$

where

$$
\begin{gathered}
m_{n}(x)=\left[\left(1-t_{n}\right) f\left(u_{n}\right)+t_{n}\left(g_{1}\left(x, u_{n}\right)-h(x)\right)\right] /\left\|u_{n}\right\|_{C^{1}} \\
+t_{n} g_{2}\left(x, u_{n}\right) /\left\|u_{n}\right\|_{C^{1}} .
\end{gathered}
$$

Clearly by (8) $\lim _{n \rightarrow \infty} m_{n}=0$ in $L^{1}(0, \pi)$. Moreover, the right hand side of $(10)$ is the sum of two terms: the first term is dominated by a function in $L^{1}(0, \pi)$ and by (7) the second term has norm in $L^{(1+p) / p}(0, \pi)$ bounded by a constant independent of $n$. Since each $v_{n}^{\prime}$ vanishes somewhere in $(0, \pi)$, it follows from (9) that the sequence $\left\{v_{n}^{\prime}\right\}$ is equicontinuous and uniformly bounded on $[0, \pi]$. Thus the sequence $\left\{v_{n}\right\}$ is also equicontinuous and uniformly bounded on $[0, \pi]$. By the Ascoli theorem we may assume without loss of generality that $t_{n} \rightarrow t_{0}$ and there exists $v \in C^{1}[0, \pi]$ such that $v_{n} \rightarrow v$ in $C^{1}[0, \pi]$. It follows from (9) that for $x \in[0, \pi]$

$$
v_{n}^{\prime}(x)=v_{n}^{\prime}(0)-\int_{0}^{x}\left[v_{n}(s)-m_{n}(s)\right] d s
$$

and so by letting $n$ approach $\infty$

$$
v^{\prime}(x)=v^{\prime}(0)-\int_{0}^{x} v(s) d s
$$

Hence $v$ has an absolutely continuous derivative and satisfies

$$
v^{\prime \prime}+v=0 \quad \text { in }(0, \pi), \quad v(0)=v(\pi)=0 .
$$

Consequently $v=\alpha \sin x$ for some $\alpha \neq 0$. We deduce using (5) that the sequence $\left\{v_{n} / \sin x\right\}$ converges to $\alpha$ uniformly on $[0, \pi]$.

Now there are two alternatives: either $\alpha>0$ or $\alpha<0$. If the first alternative holds, then $u_{n}(x)>0$ for $n$ large enough and $\lim _{n \rightarrow \infty} u_{n}(x)=\infty$ for $x \in(0, \pi)$. We write (6) with $u=u_{n}$ and $t=t_{n}$ as

$$
\left(1-t_{n}\right) \int_{0}^{\pi} f\left(u_{n}\right) \sin x d x+t_{n} \int_{0}^{\pi}\left[g\left(x, u_{n}\right)-h(x)\right] \sin x d x=0
$$

and have the following two cases to consider. In the case $t_{0}=0$, by (H) and (11)

$$
\left(1-t_{n}\right) \int_{0}^{\pi} f\left(u_{n}\right) \sin x d x \leqslant t_{n} \int_{0}^{\pi}[h(x)+b(x)] \sin x d x
$$


and so by the Lebesgue theorem we would have $\int_{0}^{\pi} \sin x d x \leqslant 0$ which is absurd. In the case $t_{0}>0$, applying the Fatou Lemma to the left hand side of (11) we would have

$$
\left(1-t_{0}\right) \int_{0}^{\pi} \sin x d x+t_{0} \int_{0}^{\pi}\left[g_{+}(x)-h(x)\right] \sin x d x \leqslant 0
$$

which contradicts the second inequality in (3). If the second alternative holds, then $u_{n}(x)<0$ for $n$ large enough and $\lim _{n \rightarrow \infty} u_{n}(x)=-\infty$ for $x \in(0, \pi)$. Again we have the following two cases to consider. In the case $t_{0}=0$, by (2) and (11)

$$
\left(1-t_{n}\right) \int_{0}^{\pi} f\left(u_{n}\right) \sin x d x \geqslant t_{n} \int_{0}^{\pi}[h(x)-c(x)] \sin x d x
$$

and so by the Lebesgue theorem we would have $-\int_{0}^{\pi} \sin x d x \geqslant 0$ which is absurd. In the case $t_{0}>0$, applying the Fatou Lemma to the left hand side of (11) we would have

$$
-\left(1-t_{0}\right) \int_{0}^{\pi} \sin x d x+t_{0} \int_{0}^{\pi}\left[g_{-}(x)-h(x)\right] \sin x d x \geqslant 0
$$

which contradicts the first inequality in (3). This completes the proof of the theorem. $]$

As pointed out in Landesman and Lazer [5], if we assume in Theorem 1 furthermore that $g_{-}(x)<g(x, u)<g_{+}(x)$ for almost all $x \in(0, \pi), u \in \mathbb{R}$, then (3) is also necessary for the solvability of (1). Thus it follows from Theorem 1 that for $p>2$

$$
\begin{gathered}
u^{\prime \prime}+u+\left(1+u_{+}^{p}\right) /\left(1+u^{2}\right)=h(x) \text { in }(0, \pi) \\
u(0)=u(\pi)=0
\end{gathered}
$$

is solvable if and only if $\int_{0}^{\pi} h(x) \sin x d x>0$, where $u_{+}=\max \{u, 0\}$.

An interesting case in which (3) is not satisfied is when the equality holds in place of one of the inequalities. By modifying part of the proof of Theorem 1, we obtain the following Theorem 2 which is an existence theorem for a solution of (1) without assuming a Landesman-Lazer condition.

Theorem 2. Let $g:(0, \pi) \times \mathbb{R} \rightarrow \mathbb{R}$ be a Caratheodory function satisfying the condition (H). If

$$
u g(x, u) \geqslant 0
$$

for almost all $x \in(0, \pi), u \in \mathbb{R}$, then the problem (1) is solvable for any $h \in L^{1}(0, \pi)$ such that $\int_{0}^{\pi} h(x) \sin x d x=0$.

Proof: In the proof of Theorem 1, the crucial part is in the second half to obtain an a priori bound in $C^{1}[0, \pi]$ for solutions $u$ to (4) for some $0 \leqslant t<1$. The condition 
(3) is used only in the final steps of this part to produce contradictions. Thus we can proceed in exactly the same way as the proof of Theorem 1 up to the point where we have two alternatives: either $\alpha>0$ or $\alpha<0$. Now (11) becomes

$$
\left(1-t_{n}\right) \int_{0}^{\pi} f\left(u_{n}\right) \sin x d x+t_{n} \int_{0}^{\pi} g\left(x, u_{n}\right) \sin x d x=0 .
$$

If the first alternative holds, then $u_{n}>0$ in $(0, \pi)$ for $n$ large enough. Since $\int_{0}^{\pi} f\left(u_{n}\right) \sin x d x>0, t_{n} \neq 0$. Thus $0<t_{n}<1$ and so by (13) we would have $\int_{0}^{\pi} g\left(x, u_{n}\right) \sin x d x<0$ which contradicts (12). Similarly the second alternative leads to a contradiction. This completes the proof of the theorem.

As an example in which (3) is not satisfied, it follows from Theorem 2 that for $p \geqslant 0$, the problem

$$
u^{\prime \prime}+u+u_{+}^{p}|\sin u|=h(x) \quad \text { in }(0, \pi), \quad u(0)=u(\pi)=0
$$

is solvable if $\int_{0}^{\pi} h(x) \sin x d x=0$.

From the proofs of Theorems 1 and 2, we see that the condition (3) is formed by two inequalities which are used in the proof separately. Likewise the condition (12) is formed by two inequalities, namely $g(x, u) \geqslant 0$ for $u \geqslant 0$ and $g(x, u) \leqslant 0$ for $u \leqslant 0$ for almost all $x \in(0, \pi)$, which are used separately. Hence one in (3) may be combined with the other in (12) to produce new solvability conditions. For example, taking into consideration the remark following the proof of Theorem 1, it follows that for $p>0$, the problem

$$
u^{\prime \prime}+u+u_{+}^{p}=h(x) \text { in }(0, \pi), \quad u(0)=u(\pi)=0
$$

is solvable if and only if $\int_{0}^{\pi} h(x) \sin x d x \geqslant 0$.

\section{REFERENCES}

[1] S. Ahmad, 'A resonance problem in which the nonlinearity may grow linearly', Proc. Amer. Math. Soc. 92 (1984), 381-384.

[2] K. Deimling, Nonlinear functional analysis, (Springer-Verlag, Berlin, Heidelberg, New York, 1985).

[3] P. Drabek, 'On the resonance problem with nonlinearity which has arbitrary linear growth', J. Math. Anal. Appl. 127 (1987), 435-442.

[4] R. Iannacci and M.N. Nkashama, 'Nonlinear two point boundary value problems at resonance without Landesman-Lazer condition', Proc. Amer. Math. Soc. 106 (1989), 943-952.

[5] E.M. Landesman and A.C. Lazer, 'Nonlinear perturbations of linear elliptic boundary value problems at resonance', $J$. Math. Mech. 19 (1970), 609-623.

Department of Mathematics

National Tsing Hua University

Hsinchu

Taiwan
Department of Banking and Insurance

Takming College of Commerce

Tajpei

Tajwan 